\section{Längere OP-Zeiten, mehr thromboembolische Komplikationen}

Sakran JV, Ezzeddine H, Haut ER et al. Prolonged operating room time in emergency general surgery is associated with venous thromboembolic complications. Am J Surg 2019; 218: 836-841

Venöse Thromboembolien (VTE) gehören zu den am ehesten verhinderbaren Ursachen für Morbidität und Mortalität bei hospitalisierten Patienten in den Vereinigten Staaten und sind besonders häufig in der Folge von Operationen. Gleichzeitig nehmen notfallmäßige Operationen in der Allgemeinchirurgie in den letzten Jahren zu. Die hohe Rate von thromboembolischen Komplikationen führt zu höheren Wiederaufnahmeraten.

Die Autoren um Sakran aus Baltimore untersuchten in ihrer retrospektiven Studie die Assoziation zwischen Operationszeit und Entwicklung von tiefen Venenthrombosen oder pulmonalen Embolien nach allgemeinchirurgischen Notfalleingriffen. Sie untersuchten dafür 6 typische Notfallprozeduren zwischen 2013 und 2015 im NSQIP-Datensatz. Mittels multivariater logistischer Regression entwickelten die Autoren Prädiktoren für die Entwicklung einer VTE.

\section{Ergebnisse}

Von 108954 Patienten entwickelten 1366 Patienten (1,3\%) eine VTE. Die mediane Zeit bis zur Diagnose lag bei 9 Tagen für die tiefe Venenthrombose und 8 Tagen für die Lungenembolie. Die Patienten wurden in 2 Gruppen eingeteilt: OP-Zeit bis 100 Minuten und OP-Zeit $\geq 100$ Minuten. Eine Operationszeit von 100 Minuten oder mehr war assoziiert mit erhöhtem Risiko für die Entwicklung einer tiefen Venenthrombose (OR 1,3; 0,95\%-KI: 1,12-2,21) und einer Lungenembolie (OR: 1,25; $95 \%-K I$ 1,11-2,43) mit einem Anstieg von $7 \%$ bzw. $5 \%$ für jede weitere Dauer von 10 Minuten über 100 Minuten OP-Zeit hinaus. Andere unabhängige Prädiktoren für VTE-Komplikationen waren höheres Alter, Krebs in der Anamnese und NotfallKolektomie.

\section{FAZIT}

Eine längere OP-Zeit, so die Autoren, insbesondere mehr als 100 Minuten, war ein unabhängiger Risikofaktor für VTE-Komplikationen nach Notfalloperationen in der Allgemeinchirurgie. Dieses Ergebnis sollten Chirurgen bei der Planung des Eingriffs bedenken. Die Autoren benennen diverse Limitationen ihrer Studie, so z. B. das retrospektive Design, die Nachbeobachtung für nur 30 Tage, die fehlenden Daten zur VTE-Prophylaxe und die fehlende Unterscheidung zwischen symptomatischen und asymptomatischen Patienten.

MOR Dr. med. Benedikt Lampl, Regensburg 